\title{
ARTEMiS, cooperative efforts, and optimal short-term strategies
}

\author{
Martin Dominik $^{* \dagger}$ \\ SUPA, University of St Andrews - School of Physics \& Astronomy, North Haugh, St Andrews, \\ KY16 9SS, United Kingdom \\ E-mail: md35est-andrews.ac.uk
}

\begin{abstract}
Besides their capability for obtaining a census of sub-Neptunian planets orbiting Galactic disk or bulge stars, existing microlensing campaigns still face the chance of yielding the first discovery of an Earth-mass planet. In order to make this likely to happen in the short term, a well-coordinated effort of survey, follow-up and anomaly monitoring is required. From 2008, ARTEMiS (Automated Robotic Terrestrial Exoplanets Microlensing Search) will merge strategy and tools that have been used by PLANET and RoboNet, and make these publicly available, thereby providing an independent open platform for rapid interchange of information. Embedding its expert system into eSTAR will moreover allow communication by means of standardized protocols with robotic telescopes that form part of the Heterogeneous Telescopes Networks (HTN) consortium. Based on the capabilities, availability, commitment, and strategic preferences associated with individual observing sites, and taking into account data-sharing agreements, ARTEMiS will be able to recommend the optimal target to be observed at any given time. The SIGNALMEN anomaly detector, an integral part, has already demonstrated its power in 2007 , where crucial data on several planet candidate events have been taken as response to notifications by its alert mechanism. The up-to-the-minute visualization and interpretation of ongoing events not only guides observers, but moreover offers an excellent opportunity for impactful public outreach, allowing the live communication of discoveries.
\end{abstract}

The Manchester Microlensing Conference: The 12th International Conference and ANGLES Microlensing Workshop

January 21-25 2008

Manchester, $U K$

\footnotetext{
*Speaker.

${ }^{\dagger}$ Royal Society University Research Fellow
} 


\section{Short-term goals for microlensing planet detection}

Various strategies have been proposed for advanced microlensing planet searches that can be realized in some years' time [1]-3]. The chances of attracting funding for such efforts however crucially depend on the ability to demonstrate an impressive record from ongoing research activities. It is therefore important to have a close look at how the most can be achieved from the resources that are currently available or will be deployed within the next $2-3$ years.

Given that more than 250 planets orbiting stars other than the Sun are already known [ 5 , just detecting further ones comes along with a subsequently decreasing scientic value and public interest attached to. Hence, in order to remain at the forefront of science, one needs to aim higher. About two years ago, the announcement of the discovery of OGLE-2005-BLG-390Lb [6], the most Earth-like extra-solar planet known at that time, manifested a relevant role in the study of terrestrial planets for the technique of gravitational microlensing. While it provided a first hint for such being common in the Universe, other planet detection techniques have meanwhile become sensitive to this regime [7-9], too. In this extremely vivid field of research, the utmost is currently done on improving on their capabilities, which means that the technologic advance of gravitational microlensing on some of its current niches is shrinking.

Currently, there are two promising short-term goals. The first is to provide a meaningful census of planets orbiting stars at galactic distances by 2010, explicitly including such whose mass and/or orbit put them beyond reach of other techniques. This would make a strong case of support for future projects that aim at observing terrestrial planets and their atmospheres, and provide otherwise unobtainable constraints on models of planet formation and orbital migration, allowing us to create a consistent picture of the origin of planets. The other one is to come up with other spectacular firsts, such as going towards lower masses or finding planets in wider orbits.

\section{Boosting the planet detection efficiency}

The discovery of OGLE-2005-BLG-390Lb [6] impressively demonstrated that we are not far at all from detecting an Earth-mass planet. Had such been in the same spot, an immediate dense anomaly monitoring after a first trigger could have revealed it, provided the availability of suitable telescopes. However, the successful implementation of such a three-step strategy of survey, followup, and anomaly monitoring requires 1.5 -hourly follow-up monitoring on a sufficient number of targets, the interchange of information, modelling, and identification of ongoing anomalies in real time, as well as activation of anomaly monitoring on the time-scale of 5-10 min.

Substantially more planets could be detected by catching all the opportunities that are provided by the collected data, of which many are currently missed. The most prominent case is OGLE2002-BLG-055 [10], which shows a clear deviation, not giving any reason to doubt that a real effect has been observed. This microlensing anomaly is not only compatible with a planet orbiting the lens star, but such an interpretation even is the most plausible one. However, any proper claim is vetoed by the lack of firm evidence, which could have arisen from immediate further observations. Consequenly, the study of this case provided motivation for OGLE to implement its Early-EarlyWarning-System (EEWS) [11]. 

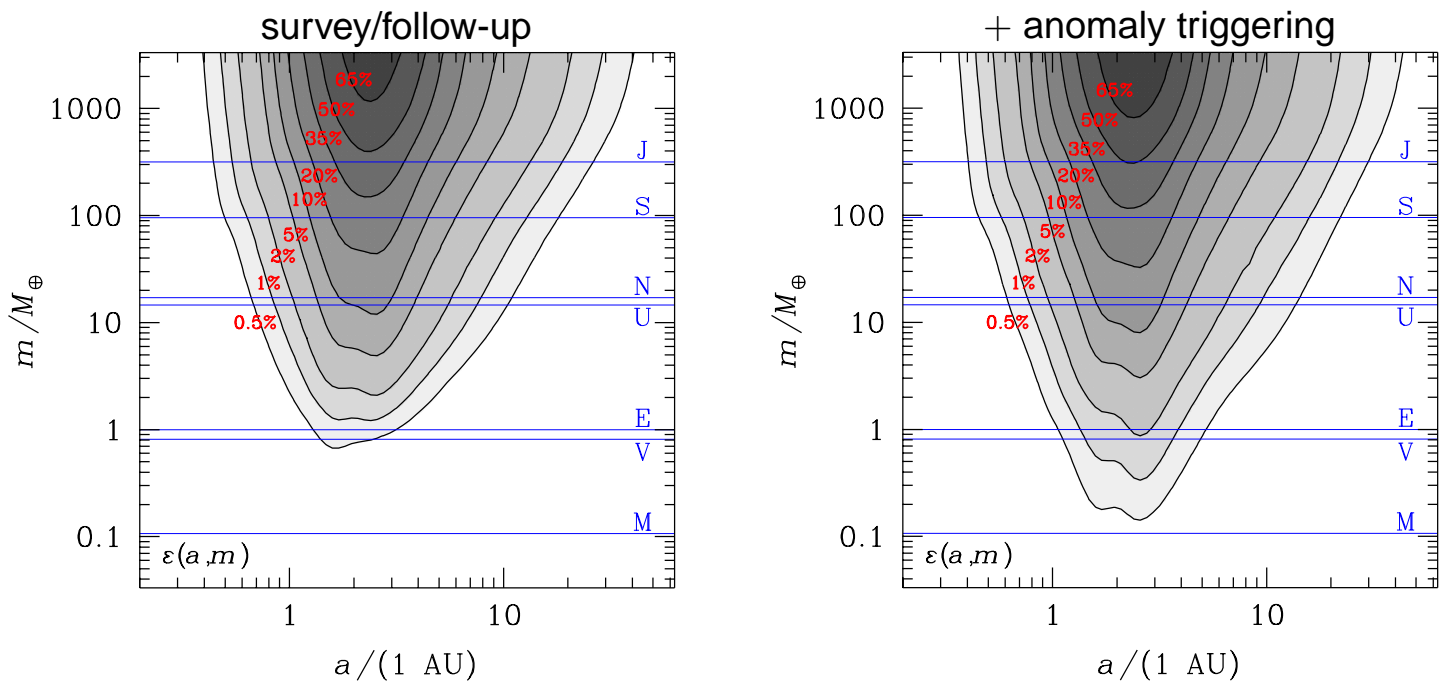

Figure 1: Pushing the planet detection efficiency of a survey/follow-up microlensing planet search towards and below Earth mass by means of high-cadence sampling triggered by an automated anomaly detector. The figures show the respective detection efficiencies $\varepsilon$ as a function of the orbital radius $a$ (assuming a circular orbit) and the mass $m$ of the planet for a microlensing event with angular Einstein radius $\theta_{\mathrm{E}}=274 \mu$ as and proper motion $\mu=13.7 \mu$ as $^{-1}$, yielding an event time-scale $t_{\mathrm{E}}=\theta_{\mathrm{E}} / \mu \sim 20 \mathrm{~d}$, assuming a kinematic model of the Milky Way and a lens star mass function [12, 13]. The adopted $\rho_{\star}=\theta_{\star} / \theta_{\mathrm{E}}=0.002$ corresponds to a main-sequence star with radius $R_{*}=D_{\mathrm{S}} \theta_{\star} \sim 1 R_{\odot}$ in the Galactic bulge at a distance $D_{\mathrm{S}} \sim 8.5 \mathrm{kpc}$. For the closest angular separation $u_{0} \theta_{\mathrm{E}}$ between lens and source star, $u_{0}=0.3$ has been assumed, which results in a peak magnification $A_{0} \sim 3.5$. The detection of a planet has been defined as observing a sequence of consecutive points on the same side of the model light curve of which at least 5 deviate by more than twice their photometric uncertainty, assumed to be $3 \%$ at baseline with a further systematic error of $0.5 \%$ added in quadrature. For the sampling intervals, $\Delta t=1 \mathrm{~d}$ has been chosen for the surveys, $\Delta t=(90 \mathrm{~min}) A^{-1 / 2}$ for follow-up observations, starting at a magnification $A=1.5$ and stopping at $A=1.06$, and $\Delta t=5 \mathrm{~min}$ for anomaly monitoring, activated after a data point is found to deviate by more than $2 \sigma$.

Apart from such obvious signals, there are also plenty of rather subtle deviations, some of which may well constitute false alerts, but others may indeed be missed opportunities instead. One should therefore avoid being too conservative on following up on potential deviations. In fact, one can live with a substantial number of false alerts as long as the time invested in further monitoring of the respective events results in an increase of the total resulting detection efficiency. On the other hand, an anomaly detection process that does not deliver any false alerts, is terribly inefficient rather than close to optimal. Opportunities are also missed if collected data are not available in real time, which prevents an immediate response - while such could turn out to be more valuable than all the other observations over the whole season together.

As illustrated in Fig. 1 1 by means of results arising from a simple simulation of an event on a main-sequence source star that reaches a peak magnification of $A_{0} \sim 3.5$, immediate intensification of monitoring on the first suspicion of a deviation allows to extend the sensitivity further down in mass by factors between 3 and 5, depending on the details of the adopted observing strategy - and Earth is no longer the limit. 


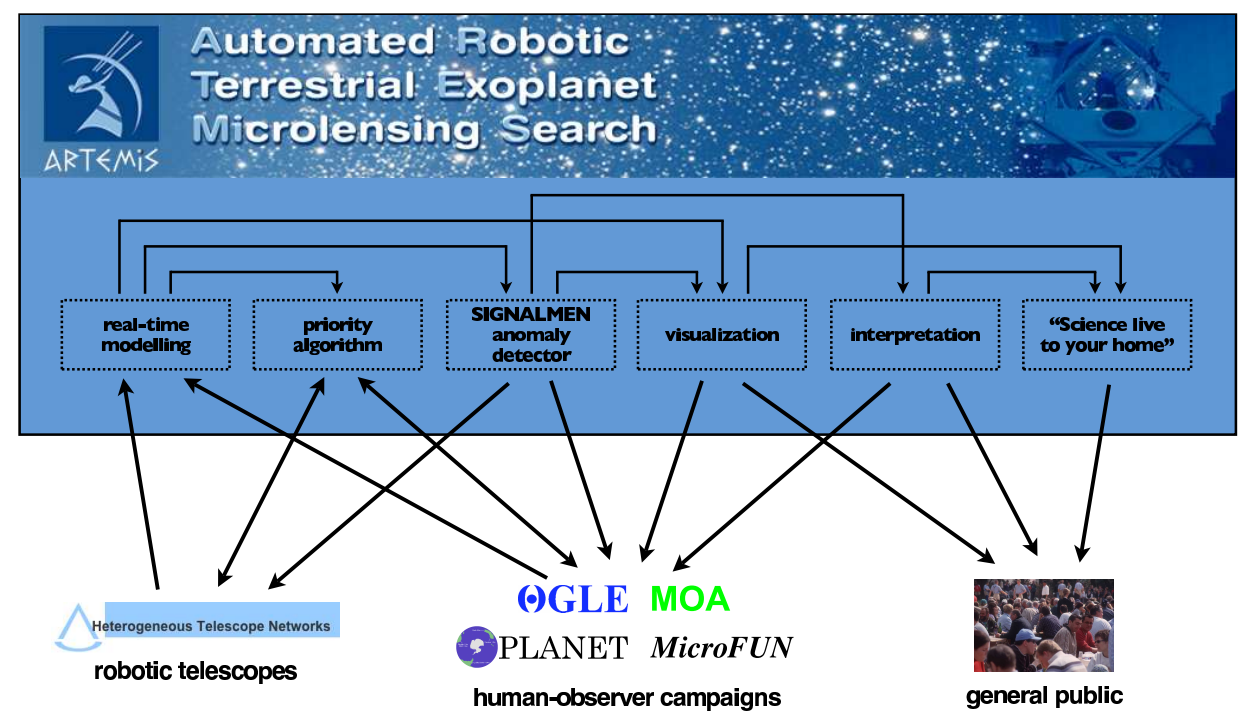

Figure 2: The components of ARTEMiS (Automated Robotic Terrestrial Exoplanet Microlensing Search) and their interaction with the outside world.

\section{The ARTEMiS concept: innovative, cooperative, and communicative}

With an innovative, cooperative, and communicative concept design, ARTEMiS (Automated Robotic Terrestrial Exoplanet Microlensing Search) [14] enables a community effort to hunt for extra-solar planets of Earth mass and below by means of the provision of an expert system for target selection, aiming at optimizing the scientific return of the globally available resources [15]. Together with RoboNet(-II), a microlensing follow-up observing campaign using a world-wide network of robotic telescopes [16], and eSTAR (e-Science Telescopes for Astronomical Research) [17], handling the scheduling by means of intelligent agents, ARTEMiS forms part of a symphony of current UK-led efforts related to microlensing planet detection. Rather than being a closed collaboration, it provides a coordinated platform for complementary individual contributions, and welcomes any good ideas that match its goals, strategy, and philosophy.

Based on the interaction of various software components and some further manual tasks, as shown in Fig. 2, ARTEMiS provides a range of services, which are provided under the sole formal condition of proper referencing and the expectation of being valued. ARTEMiS is to manage interfaces that allow to communicate both with robotic telescopes, where it is intended to talk to sites within the Heterogeneous Telescope Networks consortium by means of the eSTAR software, and with human-observer sites. Furthermore, it contains an outreach component for gravitational microlensing that engages in a dialogue with the general public and communicates forefront scientific research just in the moment it is being carried out. ARTEMiS will specifically provide real-time models of events, site-tailored recommendations for follow-up on ordinary events and for checking on potential anomalies, alerts on identified ongoing anomalies, real-time plots showing current data and model light curves, and information about the nature of ongoing anomalies. In particular, the most recently data from any specified site(s) along with a model light curve can be followed almost live just by pointing a web-browser to the respective cgi-script. 


\section{SIGNALMEN: Real-time modelling and anomaly detection}

The SIGNALMEN anomaly detector [18] forms an integral part of the ARTEMiS expert system. Since anomalies can only be detected against suitable models of ordinary light curves from which they are suspected to depart, a sophisticated modelling agent operates in the SIGNALMEN core. Apart from it being multi-site capable, it uses maximum a-posteriori estimation of parameters [19], and applies a robust-fitting algorithm with relative weighting between observing sites. These features leave the modelling core and anomaly assessment of SIGNALMEN with very little vulnerability to data outliers and large scatter in the data, and moreover ensure the provision of an as-accurate-as-possible picture of the nature of ongoing events at any time.

As compared to the OGLE EEWS, SIGNALMEN has been designed to work in a different environment, which sets different requirements, and calls for different solutions. While considering the data inhomogeneity arising from the different sites, it also accounts for the fact that no observer is present. In particular, this implies that a single data point cannot lead to any meaningful judgement. Instead, the concept of SIGNALMEN is based on the opportunity of real-time photometry and robotic operation of the telescopes allowing for an immediate feedback, where a real-time assessment of data coming from the telescopes defines which target to observe next. Based on a model of all currently available data, SIGNALMEN will come up with one out of three possible conclusions: (1) there is an ongoing anomaly, (2) there is no ongoing anomaly, or (3) not sure what is going on. The uncertainty of the last case is simply overcome by requesting further data until a firm conclusion can be reached.

It was the declared goal for 2007 to reach a fraction of false alerts that does not significantly exceed that of PLANET homebase, which is about 10-15\%. This was achieved on the basis of 599 OGLE events assessed between April and mid-October: 34 events were correctly identified as involving ongoing anomalies; 3 further events were flagged as anomalous, whose status is unclear due to the lack of data; 2 marginal events were considered to be lacking of evidence for an anomaly; 11 events that visual inspection identifies as anomalous were not reported due to the anomaly being over at the time of data release; none of the anomalous events that should have been flagged was missed; 1 event was reported anomalous at the right time for the wrong reason (error of the third kind); and finally there were 3 false alerts (if one eliminates the cases of failed modelling that are now fixed). However, as compared to PLANET homebase, SIGNALMEN was able to provide 3 times as many alerts. Nevertheless, SIGNALMEN did not face a chance of triggering further observations on about a quarter of the anomalies, because, when the first data containing them were released, those were found not to be in progress anymore.

During the 2007 observing season, SIGNALMEN not only properly identified deviations not obvious to the human eye, such as the first sign of finite-source effects in MOA-2007-BLG233/OGLE-2007-BLG-302, but alerts circulated to observing teams resulted in crucial data on the planetary candidate events MOA-2007-BLG-197 [20] and OGLE-2007-BLG-368/MOA-2007BLG-308 [21] being collected, that would not be available otherwise. In all these cases, the combination of data from different sites allowed SIGNALMEN to unleash its power, based on the ability to perform a bunch of tests faster than any human. 


\section{Target recommendation}
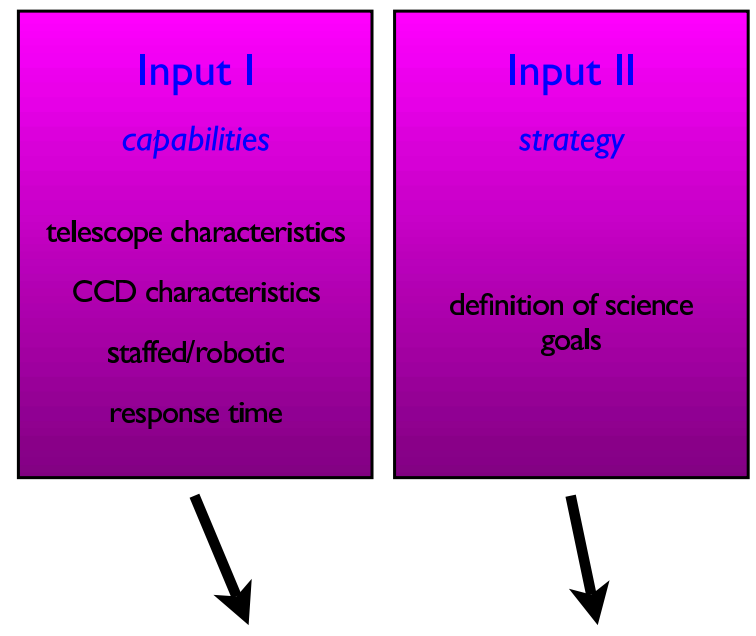
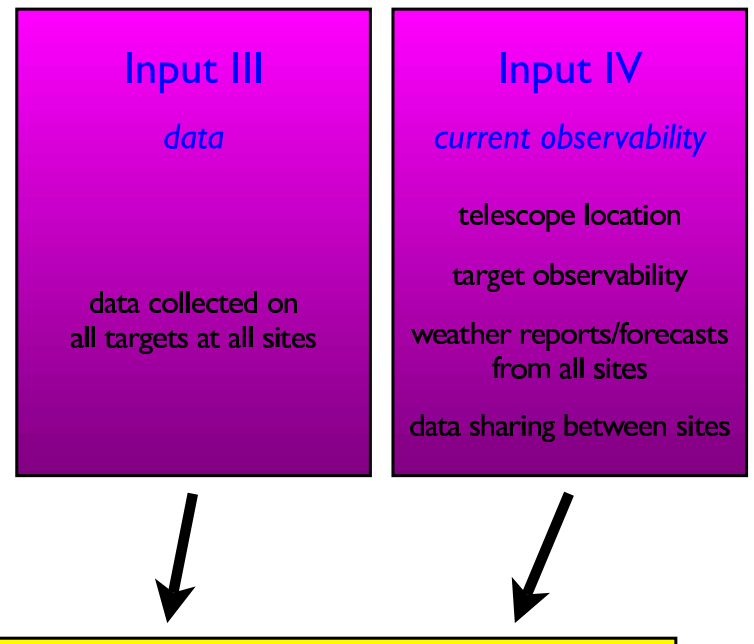

Output

optimal target to be observed by a specified site at any given time

Figure 3: Target selection by ARTEMiS, to be developed during 2008.

\section{Implementing optimal planet search strategies}

The central aim of ARTEMiS is to enable the realization of an optimal planet search strategy by serving each telescope site with the most promising target to be observed at any given time. This not only includes the recommendation of targets for anomaly monitoring following suspicion of a deviation from an ordinary light curve, but also the selection of ongoing microlensing events for follow-up observations that leads to as many detected deviations of the desired kind as possible. However, there is no single optimal global strategy. Instead, as illustrated in Fig. 目, the optimal choice of targets critically depends on 4 different kinds of input: the capabilities of potential observing sites, the science goals, the currently available data, and the current observability.

On the science goals, one needs to decide whether to go for (1) maximizing the number of planets detected, (2) determining their abundance, or (3) obtaining a census of their properties. Moreover, the strategy will differ on what kind of planets one is interested in, and whether time shall be devoted to fields of research apart from extra-solar planets. For any given set of resources, different science goals have their respective degree of achievability and their specific return for the made investment. Even for the optimization, there are at least two different ways to proceed, namely going for the largest possible return or the best return-to-investment ratio. On this issue, the strategic options currently adopted by PLANET and MicroFUN differ substantially. 


\section{Summary and conclusions}

Currently available resources could be used more efficiently, thereby substantially increasing the returns from and the visibility of exoplanet searches exploiting the technique of gravitational microlensing. ARTEMiS provides the technical means for an optimized cooperative effort and far-reaching outreach opportunities.

\section{References}

[1] A. Gould, B.S. Gaudi and D.P. Bennett, Ground-based Microlensing Surveys, arXiv: 0704.0767.

[2] P. Yock, Detecting Earth-like extra-solar planets from Antarctica by gravitational microlensing, AcASn 47 (2006) 410 [astro-ph/ 0508603 ].

[3] D.P. Bennett, I. Bond, E. Cheng, S. Friedman, P. Garnavich, B.S. Gaudi, R. Gilliland, A. Gould, M.A. Greenhouse, K. Griest, R.A. Kimble, J.I. Lunine, J.C. Mather, D. Minniti, M. Niedner, B. Paczyński, S. Peale, B.J. Rauscher, R.M. Rich, K. Sahu, D. Tenerelli, A. Udalski, N. Woolf and P. Yock, The Microlensing Planet Finder: completing the census of extrasolar planets in the Milky Way, Proc. SPIE 5487 (2004) 1453 [astro-ph/ 0409218 ].

[4] S. Udry and N.C. Santos, Statistical Properties of Exoplanets, ARA\&A 45 (2007) 397.

[5] J. Schneider, The Extrasolar Planets Encyclopaedia, http://exoplanet.eu.

[6] J.-P. Beaulieu, D.P. Bennett, P. Fouqué, A. Williams, M. Dominik, U.G. Jørgensen, D. Kubas, A. Cassan, C. Coutures, J. Greenhill, K. Hill, J. Menzies, P.D. Sackett, M. Albrow, S. Brillant, J.A.R. Caldwell, J.J. Calitz, K.H. Cook, E. Corrales, M. Desort, S. Dieters, D. Dominis, J. Donatowicz, M. Hoffman, S. Kane, J.-B. Marquette, R. Martin, P. Meintjes, K. Pollard, K. Sahu, C. Vinter, J. Wambsganss, K. Woller, K. Horne, I. Steele, D.M. Bramich, M. Burgdorf, C. Snodgrass, M. Bode, A. Udalski, M.K. Szymański, M. Kubiak,T. Wieckowski, G. Pietrzyński, I. Soszyński, O. Szewczyk, O., Ł. Wyrzykowski, B. Paczyński, F. Abe, I.A. Bond, T.R. Britton, A.C. Gilmore, J.B. Hearnshaw, Y. Itow, K. Kamiya, P.M. Kilmartin, A.V. Korpela, K. Masuda, Y. Matsubara, M. Motomura, Y. Muraki, S. Nakamura, C. Okada, K. Ohnishi, N.J. Rattenbury, T. Sako, S. Sato, M. Sasaki, T. Sekiguchi, D.J. Sullivan, P.J. Tristram, P.C.M. Yock and T. Yoshioka, Discovery of a cool planet of 5.5 Earth masses through gravitational microlensing, Nature 439 (2006) 437 [astro-ph/0601563].

[7] P. Bordé, D. Rouan and A. Léger, Exoplanet detection capability of the COROT space mission, A\&A 405 (2003) 1137 [astro-ph / 0305159 ].

[8] S. Udry, X. Bonfils, X. Delfosse, T. Forveille, M. Mayor, C. Perrier, F. Bouchy, C. Lovis, F. Pepe, D. Queloz and J.-L. Bertaux, The HARPS search for southern extra-solar planets. XI. Super-Earths (5 and $8 M_{\oplus}$ ) in a 3-planet system, A\&A 469 (2007) L43 [arXiv: 0704 . 3841].

[9] F. Selsis, B. Chazelas, P. Bordé, M. Ollivier, F. Brachet, M. Decaudin, F. Bouchy, D. Ehrenreich, J.M. Grießmeier, H. Lammer, C. Sotin, O. Grasset, C. Moutou, P. Barge, M. Deleuil, D. Mawet, D. Despois, J.F. Kasting and A. Léger, Could we identify hot ocean-planets with CoRoT, Kepler and Doppler velocimetry?, Icarus 191 (2007) 453 [astro-ph/ 0701608 ].

[10] M. Jaroszyński and B. Paczyński, A Possible Planetary Event OGLE-2002-BLG-055, AcA 52 (2002) 361 [astro-ph/0212023].

[11] A. Udalski, The Optical Gravitational Lensing Experiment. Real Time Data Analysis Systems in the OGLE-III Survey, AcA 53 (2003) 291 [ast ro-ph/ 0401123 ]. 
[12] M. Dominik, Stochastic distributions of lens and source properties for observed galactic microlensing events, MNRAS 367 (2006) 669 [astro-ph/ 0507540 ].

[13] D. Kubas, A. Cassan, M. Dominik, D.P Bennett, J. Wambsganss, S. Brillant, J.P. Beaulieu, M.D. Albrow, V. Batista, M. Bode, D.M. Bramich, M. Burgdorf, J.A.R. Caldwell, H. Calitz, K.H. Cook, Ch. Coutures, S. Dieters, D. Dominis Prester, J. Donatowicz, P. Fouqué, J. Greenhill, K. Hill, M. Hoffman, K. Horne, U.G. Jørgensen, N. Kains, S. Kane, J.B. Marquette, R. Martin, P. Meintjes, J. Menzies, K.R. Pollard, K.C. Sahu, C. Snodgrass, I. Steele, Y. Tsapras, C. Vinter, A. Williams, K. Woller and M. Zub, Limits on additional planetary companions to OGLE-2005-BLG-390L, A\&A (2008) in press [DOI: 10.1051/0004-6361:20077449], arXiv: 0710.5306.

[14] ARTEMiS - Automated Robotic Terrestrial Exoplanet Microlensing Search, http://www.artemis-uk.org.

[15] M. Dominik, K. Horne, A. Allan, N.J. Rattenbury, Y. Tsapras, C. Snodgrass, M.F. Bode, M.J. Burgdorf, S.N. Fraser, E. Kerins, C.J. Mottram, I.A. Steele, R.A. Street, P.J. Wheatley and Ł. Wyrzykowski, ARTEMiS (Automated Robotic Terrestrial Exoplanet Microlensing Search): A possible expert-system based cooperative effort to hunt for planets of Earth mass and below, AN 329 (2008) 248 [arXiv: 0801.2162$].$

[16] M.J. Burgdorf, D.M. Bramich, M. Dominik, M.F. Bode, K.D. Horne, I.A. Steele, N. Rattenbury and Y. Tsapras, Exoplanet detection via microlensing with RoboNet-1.0, P\&SS 55 (2007) 582.

[17] I.A. Steele, T. Naylor, A. Allan, J. Etherton and C.J. Mottram, eSTAR: a distributed telescope network, in Advanced Global Communications Technologies for Astronomy II, Proc. SPIE 4845 (2002) 13.

[18] M. Dominik, N.J. Rattenbury, A. Allan, S. Mao, D.M. Bramich, M.J. Burgdorf, E. Kerins, Y. Tsapras and $Ł$. Wyrzykowski, An anomaly detector with immediate feedback to hunt for planets of Earth mass and below by microlensing, MNRAS 380 (2007) 792 [arXiv: 0706.2566 ].

[19] M.D. Albrow, Early Estimation of Microlensing Event Magnifications, ApJ 607 (2004) 821 [astro-ph/0402323].

[20] A. Cassan, Preliminary results on planetary candidate MOA-2007-BLG-197, in proceedings of The Manchester Microlensing Conference: The 12th International Conference and ANGLES Microlensing Workshop, PoS (GMC8) 042 .

[21] T. Sumi, MOA-II microlensing survey, in proceedings of The Manchester Microlensing Conference: The 12th International Conference and ANGLES Microlensing Workshop, BoS (GMC8) 025. 